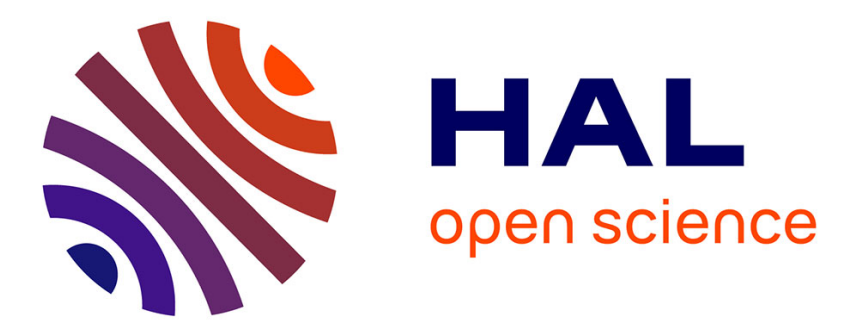

\title{
Stochastic replay of non-WSSUS underwater acoustic communication channels recorded at sea
}

François-Xavier Socheleau, Christophe Laot, Jean-Michel Passerieux

\section{To cite this version:}

François-Xavier Socheleau, Christophe Laot, Jean-Michel Passerieux. Stochastic replay of non-WSSUS underwater acoustic communication channels recorded at sea. IEEE Transactions on Signal Processing, 2011, 59 (10), pp.4838 - 4849. hal-00640755

\section{HAL Id: hal-00640755 https://hal.science/hal-00640755}

Submitted on 10 Jun 2021

HAL is a multi-disciplinary open access archive for the deposit and dissemination of scientific research documents, whether they are published or not. The documents may come from teaching and research institutions in France or abroad, or from public or private research centers.
L'archive ouverte pluridisciplinaire HAL, est destinée au dépôt et à la diffusion de documents scientifiques de niveau recherche, publiés ou non, émanant des établissements d'enseignement et de recherche français ou étrangers, des laboratoires publics ou privés. 


\title{
Stochastic Replay of non-WSSUS Underwater
}

\section{Acoustic Communication Channels Recorded}

\author{
at Sea \\ Francois-Xavier Socheleau*‡, Student Member, IEEE, Christophe Laot*, Member, IEEE, and \\ Jean-Michel Passerieux $\ddagger$ \\ *Institut Telecom; Telecom Bretagne; UMR CNRS 3192 Lab-STICC, \\ Université européenne de Bretagne \\ Technopôle Brest Iroise-CS 83818, 29238 Brest Cedex, France \\ Email: \{fx.socheleau, christophe.laot\}@telecom-bretagne.eu \\ $\ddagger$ Thales Underwater Systems, 525 route des Dolines, BP 157, 06903 Sophia Antipolis Cedex, \\ France \\ Email: jean-michel.passerieux@fr.thalesgroup.com
}

\begin{abstract}
To fully exploit sea experiments under controlled and reproducible laboratory conditions, a channel model driven by real data is derived. This model relies on the assumption that a channel recorded at sea is a single observation of an underlying random process. From this single observation, the channel statistical properties are estimated to then feed a stochastic simulator that generates multiple realizations of the underlying process. Based on the analysis of data collected in the Atlantic ocean and the Mediterranean sea, we fully relax the usual wide-sense stationary uncorrelated scattering (WSSUS) assumption. We show thanks to the empirical mode decomposition that a trend stationary model suits the analyzed underwater acoustic communication channels very well. Scatterers with different path delays are also assumed to be potentially correlated so that the true second order statistics of the channel are taken into account by our model. Test cases illustrate the benefits of channel stochastic replay to communication system design and validation. The Matlab code corresponding to the proposed simulator is available at http://perso.telecom-bretagne.eu/fxsocheleau/software.
\end{abstract}

\section{Index Terms}

Channel model, underwater acoustic communication, empirical mode decomposition 


\section{INTRODUCTION}

In order to anticipate in laboratory the performance of acoustic communication systems in real underwater environments, propagation channel models are essential. Depending on their degree of completeness and accuracy, channel models can highly increase the probability of field trial success and thus reduce the cost of overall system development. Numerous modeling techniques are available in the literature [1]-[11]. These underwater acoustic channel (UAC) models rely on formalisms that are usually either deterministic and physics-driven [4], [5], [9] or stochastic [6], [7] or a combination of both (the moments of the stochastic models being computed from physical parameters) [2], [3], [8], [10].

Channel modeling is usually faced with the dilemma of capturing the maximum of true ocean dynamic processes while limiting the number of input parameters and the computational cost. In the context of underwater acoustic communication, stochastic modeling offers a good compromise since it allows to reduce the combinations of many physical processes to only a few statistical parameters in a formalism well adapted to the communication engineering community. Stochastic modeling also appears as the most suitable manner to take into account the fast time fluctuations of the channel response due to random phenomena such as reflection on rough interfaces (sea surface and bottom), random motions of the transmitter (TX) and receiver (RX) around their nominal position, etc. In this case, the channel is modeled as a linear random time-varying system defined by its impulse response $h(t, \tau)$ so that the input $x(t)$ and the output $y(t)$ of this system satisfy [12]

$$
y(t)=\int_{-\infty}^{\infty} h(t, \tau) x(t-\tau) d \tau .
$$

The channel is then entirely characterized by the joint cumulative distribution function of the random process $h(t, \tau)$. While its marginal distributions can be well approximated by Gaussians (Rayleigh or Rice fading) in some cases [13], [14], the time fluctuations of such a process are difficult to model since they depend on phenomena of different scales (surface wave spectrum, platform motion, scatterers, seasonal cycles etc.).

To avoid ad-hoc modeling based on intuition or on difficult-to-obtain parameters, the authors in [15] recently proposed a UAC channel simulator driven by measured data. The idea relies on the postulate that a communication channel probed at a given location over a given time window is an observation of an underlying ergodic random process. Based on this single observation, the objective is then to be able to generate an infinite number of realizations of this process. This is what is called channel stochastic replay. As stated in [15], such a simulator lacks the universal applicability of usual propagation models. However, this approach is of great interest to fully exploit sea experiments under controlled and 
reproducible laboratory conditions. From an impulse response recorded at sea, it is thus possible to test a communication system in various noise conditions and for different type of modulations. It is also possible to compute fading statistics such as average level crossing rate or average fade duration [16], useful for communication system design.

To simplify the statistical description of the channel impulse response, the wide-sense stationary uncorrelated scattering (WSSUS) assumption is very often invoked. This assumption states that the channel correlation function is time-invariant and that the scatterers with different path delays are uncorrelated so that the second-order statistics of the channel are reduced from four to two dimensions [12], [17]. The WSSUS assumption is appealing since it greatly simplifies the model. The rationale for this assumption is usually that over a restricted period of time and a small bandwidth, this assumption is reasonably satisfied (quasi-WSSUS) [15], [18]. However, when manipulating real data, it can be very difficult to formally quantify the time-frequency windows over which this assumption is locally valid. This is particularly true when communicating platforms are moving. Channel impulse responses being dependent of range and depth and TX/RX motion being known to introduce correlation between the Doppler rates of different paths, experiments with moving platforms are not expected to reveal WSSUS channels.

Based on a set of measures collected at sea in a coastal environment, we analyze in this paper the statistical properties of the UAC and propose a novel channel model applied to stochastic replay. Thanks to the empirical mode decomposition [19], we fully relax the (quasi-) wide sense stationary assumption and show that the analyzed UAC are trend stationary. We also assume that the scatterers with different path delays can be correlated so that the true second order statistics of the UAC are very well approximated by our model.

The derivation of the proposed method is organized in two main parts. The first part, sections II and III, deals with the characterization and the modeling of the UAC channel. More precisely, section II describes sea experiments conducted in the Atlantic Ocean and the Mediterranean sea as well as the channel impulse response estimation methods. In section III, we infer some statistical properties of the measured UAC that are of prime importance to build our model. The second part, sections IV and V, presents the channel simulator based on the principle of stochastic replay and some of its applications. In section IV, we show how to generate new realizations of the measured channel while complying with its intrinsic statistical properties and section $\mathrm{V}$ illustrates the practical interest of such a model. Finally, conclusions are given in section VI. Additionally, the Matlab code corresponding to the proposed simulator is available at http://perso.telecom-bretagne.eu/fxsocheleau/software. 


\section{Channel Sounding}

\section{A. Sea experiments}

The channel model derived in this paper relies on the analysis of experimental data collected over years in shallow water. To illustrate the model validity, two sets of complementary data have been selected. The first set of data was collected by Thales Underwater Systems in the vicinity of Sanary-surmer (Mediterranean Sea, France) in October 2004 and the second by the GESMA (Groupes d'Etudes Sous-Marines de l'Atlantique) in the Brest harbor (Atlantic Ocean, France) in October 2007. The two experimental set-ups, as well as the trial conditions, are summarized in Table I.

TABLE I

SEA EXPERIMENTS SET-UP

\begin{tabular}{|c|c|c|}
\hline & Mediterranean Sea (2004) & Atlantic Ocean (2007) \\
\hline $\begin{array}{l}\text { Tx power }(\mathrm{dB} \text { re } 1 \mu \mathrm{Pa} @ 1 \\
\text { m) }\end{array}$ & 190 & 185 \\
\hline Tx-Rx distance range $(\mathrm{m})$ & $500-10000$ & $500-3000$ \\
\hline Water depth (m) & $60-120$ & $10-40$ \\
\hline Tx/Rx immersion (m) & $20-50 / 20-50$ & $5 / 5$ \\
\hline $\begin{array}{l}\text { Tx/Rx motion speed range } \\
\left(\mathbf{m} . \mathrm{s}^{-1}\right)\end{array}$ & $0-4$ & $0-3$ \\
\hline Carrier frequency $(\mathrm{kHz})$ & 6 & 11.2 or 17.5 \\
\hline Probe signal & $\begin{array}{l}\text { Pseudo random binary sequence } \\
\text { (PRBS) of duration } 48 \mathrm{~ms} \text { re- } \\
\text { peated every } 125 \mathrm{~ms} \text { (best possi- } \\
\text { ble binary sequence with length } \\
\mathrm{N}=48 \text {, as given in reference [ } 20 \text {, } \\
\text { pp. 293]). }\end{array}$ & $\begin{array}{l}\text { Continuous QPSK modulated } \\
\text { vocoded data flow at baudrates } \\
\text { of } 2.9 \mathrm{kBd} \text { or } 4.35 \mathrm{kBd}^{b}\end{array}$ \\
\hline Sea surface conditions & $\begin{array}{l}\text { Wind speed } 10-15 \mathrm{~m} \cdot \mathrm{s}^{-1} \text {, mod- } \\
\text { erate wave }\end{array}$ & Calm, little swell \\
\hline
\end{tabular}

${ }^{b}$ Note that the peak sidelobe of the aperiodic correlation of this sequence is $3,{ }^{b}$ refer to [21] for more details

\section{B. Channel impulse response estimation}

The estimation method of the channel impulse response strongly depends on the kind of probe signal recorded at sea. For the trials in 2004, a filter matched to a pseudo-random binary sequence (PRBS) was implemented to provide the time evolution of the channel response. Such a probe is commonly 
used for channel sounding since its autocorrelation function approaches the unit impulse. The PRBS of the Mediterranean trial was designed to tailor the sounded channel. In fact, the transmitted probe can monitor channels with excess delay spread up to $125 \mathrm{~ms}$, with a $1 \mathrm{~ms}$ delay resolution. The channel response estimates are updated eight times per second which allows to track channels with relatively small coherence time ${ }^{1}$. In 2007, the experiment was not originally designed for channel sounding so that no dedicated probe signal was used. However, since all the transmitted data were perfectly known at reception, it was possible to provide the channel state information by least mean square data-aided (LMSDA) adaptive channel estimation. The main advantage of this method, as opposed to the PRBS matched filter, is that there is less compromise to make between the ability to measure long excess delay spread and fast varying channel. For instance, the implemented algorithm can track channels with a coherence time of the order of several tens of QPSK symbol periods without theoretical limit on the excess delay spread. However, the drawback of this method lies in the difficulty to tune the LMS algorithm to avoid smoothed or noisy estimates.

In order to study and model the intrinsic properties of the UAC, TX/RX motion compensation is required prior to channel estimation. The relative velocity between the two communication endpoints indeed induces a time-varying Doppler compression/expansion that can obscure the true channel Doppler spread [15] and that causes the multipath arrivals to drift in the delay domain. Figure 1-(a) shows an example of a time-varying drift in the delay domain due to platform motion. The average drift is approximately $8 \mathrm{~ms}$ over $20 \mathrm{~s}$ which corresponds to relative velocity between TX and RX of $0.6 \mathrm{~m} . \mathrm{s}^{-1}$. The channel is assumed to have the same Doppler scale on all paths so that the multipath time drift can be mitigated by resampling of the recorded signal. When using a PRBS, the resampling factor is iteratively assessed by a bank of Doppler-shifted signal replicas combined with the phase tracking of the most energetic tap [15]. In the case of continuous QPSK data flow, the resampling procedure is based on an open-loop scheme [22] for coarse correction and on a closed-loop [23] for fine time recovery.

In the channel response estimation procedure, we implicitly assume that the probe signal is bandlimited to a bandwidth $B$ and that the power spectral density of the channel time fluctuations has a bounded support of width $f_{d}$ with $f_{d} \leq B$ so that the channel can be represented by its discrete time baseband equivalent model

$$
g_{l}(k)=\int_{-\infty}^{\infty} h\left(k T_{s 1}, \tau\right) e^{-i 2 \pi f_{c} \tau} \operatorname{sinc}\left(\frac{\tau-l T_{s 2}}{T_{s 2}}\right) d \tau
$$

$g_{l}(k)$ is the output of the channel estimation representing the $k$-th sample of the $l$-th channel tap, $f_{c}$ the

\footnotetext{
${ }^{1}$ Note that update period of the channel response estimation is bounded by the PRBS sequence duration
} 
carrier frequency and $0 \leq l \leq L$ for finite channel delay spread. $T_{s 1}$ and $T_{s 2}$ denote the sampling period in the time and delay domain respectively with $T_{s 1} \leq 1 / f_{d}$ and $T_{s 2} \leq 1 / B$.

Figure 2 shows some examples of measured channel response (after Doppler compensation) during the 2004 and 2007 experiments for various transmission ranges $D$. For the Mediterranean sea trials, $T_{s 1}=125 \mathrm{~ms}, T_{s 2}=166 \mu \mathrm{s}$ and the total observation duration is limited to $30 \mathrm{~s}$. For the signals recorded in the Atlantic Ocean, $T_{s 1}=9$ or $14 \mathrm{~ms}, T_{s 2}=115$ or $170 \mu \mathrm{s}$, and the observation duration is limited to $160 \mathrm{~s}$. Both sets of experiments were realized in good SNR conditions which guarantees accurate channel estimates. Excess delay spread ranges from 10 to $15 \mathrm{~ms}$ for Atlantic channels and from 50 to $60 \mathrm{~ms}$ for Mediterranean channels. For both experiments, it can be noticed that short range channels show a predominant path that does not fluctuate much over the time.

\section{STATISTICAL INFERENCE}

\section{A. Trend stationary channel model}

As discussed in the introduction, the channel wide sense stationary (WSS) assumption is often admitted to simplify models. It states that for all $0 \leq l \leq L$ and for all $k, k_{1}$ and $k_{2} \in \mathbb{Z}$

$$
\begin{aligned}
\mathbb{E}\left[g_{l}\left(k_{1}\right)\right] & =\mathbb{E}\left[g_{l}\left(k_{2}\right)\right], \\
\mathbb{E}\left[\left|g_{l}\left(k_{1}\right)\right|^{2}\right] & =\mathbb{E}\left[\left|g_{l}\left(k_{2}\right)\right|^{2}\right]<\infty, \\
\mathbb{E}\left[g_{l}\left(k_{1}\right) g_{l}^{*}\left(k_{2}\right)\right] & =\mathbb{E}\left[g_{l}(k) g_{l}^{*}\left(k+k_{2}-k_{1}\right)\right] .
\end{aligned}
$$

While the concept is well-defined in theory, testing stationarity on real data is not straightforward. Stationarity refers to a strict invariance of statistical properties over time, but common practice generally considers this invariance in a looser sense, relative to some observation scale [24]. In the following, we therefore refer to as stationarity relative to the observation scale.

By looking at the envelope of the measured UACs, we can infer some properties with regard to channel stationarity. For instance, Figure 3 displays the time evolution of the envelope of 3 different taps belonging to the channel (f) of Figure 2. It suggests that signals propagating through a UAC are affected by fading phenomena of different time scales. Fading is usually qualified as slow or fast to refer to the rate at which the magnitude and phase of the channel fluctuate compared to the delay requirement of the application that uses the channel (e.g. coherence time vs codeword duration). Fast fading is predominant in the design of communication systems since the adaptive algorithms at reception must be tuned to its characteristics to ensure good performance. Slow fading only represents a long-term variation of the signal-to-noise ratio whose impact on the reception performance is usually well known analytically [25]. 
Figure 3 indicates that the WSS assumption is not verified over the observation scale since the local mean of the taps envelopes is time-variant. However, the second order statistics of these envelopes seem to be invariant. These observations suggest two modeling assumptions

- (A1): Slow and fast fading are combined in an additive way.

- (A2): The UAC is a trend stationary random process so that each tap satisfies

$$
g_{l}(k)=d_{l}(k)+w_{l}(k)
$$

with, for all $k, k_{1}$ and $k_{2} \in \mathbb{Z}$

$$
\mathbb{E}\left[g_{l}(k)\right]=d_{l}(k),
$$

and

$$
\begin{aligned}
\mathbb{E}\left[\left(g_{l}\left(k_{1}\right)-\mathbb{E}\left[g_{l}\left(k_{1}\right)\right]\right)\left(g_{l}\left(k_{2}\right)-\mathbb{E}\left[g_{l}\left(k_{2}\right)\right]\right)^{*}\right] & =\mathbb{E}\left[w_{l}\left(k_{1}\right) w_{l}^{*}\left(k_{2}\right)\right] \\
& =\mathbb{E}\left[w_{l}(k) w_{l}^{*}\left(k+k_{2}-k_{1}\right)\right]
\end{aligned}
$$

$d_{l}(k)$ is called the trend which is a pseudo-coherent component that behaves almost as if the medium was deterministic and $w_{l}(k)$ is a zero-mean WSS ergodic random process. $d_{l}(k)$ can be interpreted as the contribution of pseudo-deterministic physical phenomena on channel fluctuations. At our observation scale (30 to 160 seconds), these phenomena are mainly due to range and depth changes, leading to time-varying dispersion/absorption loss, shadowing due to the topology of the environment, or to wave undulation. In agreement with $(\mathbf{A 1}), d_{l}(k)$ can be seen as the component contributing to slow fading. As for $w_{l}(k)$, it represents the channel fluctuations imputable to the scatterers that result in fast fading. Note that $d_{l}(k)$ is here considered as pseudo-deterministic only because the observation window is time-limited compared to the fluctuation speed of the underlying physical phenomena. A longer observation window may lead to different conclusions. Once again, statistical analyses are related to the observation scale. In addition, note that the concept of trend does not necessarily mean that $\left|d_{l}(k)\right|$ is affine and/or monotonic.

To validate and define more precisely our trend stationary model, we now aim at isolating the trend from the purely random process. If we succeed in isolating these two components and getting consistent properties on $d_{l}(k)$ and/or $w_{l}(k)$ over the various measured channel responses, we will thus show that (A1) and (A2) are meaningful assumptions. To find an operator or a space in which the components are separable, we focus on their physical properties. According to (A2), $d_{l}(k)$ is driven by slow varying phenomena that lead to fading fluctuations over a period of a few seconds (for waves undulation for instance) to several minutes (platform drift) whereas the scatterers induce fading with a coherence 
time usually in the order of tens to hundreds of milliseconds. Consequently, $d_{l}(k)$ and $w_{l}(k)$ can be discriminable thanks to their respective magnitude. The difficulty is then to filter complex-valued channel taps $g_{l}(k)$ based on their module fluctuation period while keeping their phase information. In fact, the phase of a channel tap does not necessarily vary at the same pace as the module and the spectrum of $d_{l}(k)$ may overlap the spectrum of $w_{l}(k)$. This suggests that classical linear filtering operators commonly used in harmonic analysis/modeling may not suit our problem very well. Another approach intuited by the observation of taps with a powerful trend (see Figure 4 for instance) is to model the trend and consequently the tap as the sum of AM-FM modulated modes.

The decomposition of non stationary multicomponent signals in "intrinsic" AM-FM contributions is possible thanks to the Empirical Mode Decomposition (EMD) introduced by Huang in [19] and briefly presented in Appendix A. EMD is appealing to solve our problem primarily because the AM-FM mode decomposition is appropriate to the analysis of non-stationary signals but also because it is data-driven and does not required any predetermined basis functions. In addition, it makes no assumption about the harmonic nature of oscillations, and can thus guarantee a compact representation (i.e., with fewer modes than a Fourier or wavelet decomposition). The method being initially limited to real-valued time series, we here use the extension to bivariate (or complex-valued) time series proposed in [26].

Each channel tap is therefore represented in the empirical mode space as

$$
g_{l}(k)=\sum_{q=0}^{Q_{l}-1} m_{l, q}(k)+r_{l}(k)
$$

where $m_{l, q}$ is the $q$-th of the $Q_{l}$ modes resulting from the EMD of the tap $g_{l}$ and $r_{l}$ is the decomposition residue. Since the fast (resp. slow) rotating modes are contributors to fast (resp. slow) fading, we then segregate the trend from the random component in the empirical modes space such that

$$
g_{l}(k)=\underbrace{\sum_{q=0}^{S_{l}-1} m_{l, q}(k)}_{w_{l}(k)}+\underbrace{\sum_{q=S_{l}}^{Q_{l}-1} m_{l, q}(k)+r_{l}(k)}_{d_{l}(k)}
$$

where $S_{l}$ is the decomposition order leading to the separation of the two components. Fading periods being measured on the signal envelope, $S_{l}$ is based on a frequency criterion computed on the modes module. For instance, if we consider that the fastest pseudo-deterministic phenomenon affecting the channels tap magnitude is the wave undulation, $S_{l}$ is chosen as the maximum order such that the average period of $\left|\sum_{q=0}^{S_{l}-1} m_{l, q}(k)\right|$ be less than the waves average period (usually in the order of few seconds). There are several ways of computing waves average period [27]. Since we are working with the module of complex-valued signals, we here consider the crest average period that is defined as the average period 
of time between the extrema of $\left|\sum_{q=0}^{S_{l}-1} m_{l, q}(k)\right|$. Note that since the EMD is an non-linear operation, the proposed filtering method is non-linear as well.

To illustrate the interest of filtering based on EMD compared to classical linear filtering, we have synthesized a signal that is the sum of an AM-FM modulated trend and a colored complex Gaussian process (see Figure 5). As shown in Figure 6, the EMD of this signal results in 7 intrinsic modes plus the residue ${ }^{2}$. This provides a compact representation of the original signal where each mode can be seen as the output of an adaptive (data-driven) time-variant filter. The trend and the random process are then rebuilt individually from the intrinsic modes. To isolate both components, the average period of $\left|\sum_{q=0}^{S_{l}-1} m_{l, q}(k)\right|$ is lower-bounded to $5 \mathrm{~s}$ which corresponds to the minimum average period of wave undulation observed in our set of experiments. This method gives $S_{l}=5$ which provides fairly accurate estimates since, for instance, the normalized mean square estimation error (NMSE) of the random component is around $-15 \mathrm{~dB}$, whereas the trend is 150 times more powerful than this random component. As a comparison, a common "linear" approach to estimate signals trend is to perform low-pass filtering using a moving average operator [14], [29]. A moving average requires an a priori knowledge on the time scale of assumed stationarity. As opposed to the EMD approach that requires some knowledge of the fading periods based on explicit physical phenomena (choice of $S_{l}$ with respect to the wave average period for instance), the predetermined time scale used for the moving average has usually little rational or physical basis and has a profound impact on the results that follow. In the example of Figure 6, the NMSE of the random component estimated by moving average ranges from -10 to $+25 \mathrm{~dB}$ for an averaging window of duration varying from $10 \mathrm{~ms}$ to $10 \mathrm{~s}$, the minimum NMSE being at $100 \mathrm{~ms}$. Moreover, a signal with the same amplitude modulation index but with a different frequency modulation index would result in a different optimal window duration. This indicates that for each analyzed channel, a different window may have to be used, which, in addition to the fact that linear filtering cannot segregate signals with overlapping spectra, highlights the idea that using linear operators to analyze trend stationary channels may not be a stable and robust option.

An example of EMD filtering applied to real data is presented in Figure 7. It shows the time evolution of a tap of the channel (f) plotted on Figure 2. The result of decomposition is in good agreement with the assumption (A2) since $w_{l}(k)$ seems here to be a realization of a zero-mean WSS process. Other examples of real tap filtering using the EMD are available http://perso.telecom-bretagne.eu/fxsocheleau/software.

\footnotetext{
${ }^{2}$ To get this result, the default values of the EMD stopping criterion detailed in [28] have been used.
} 


\section{B. Channel distribution modeling}

Thanks to EMD filtering, it is now possible to study the statistical characteristics of the complexvalued random component $w_{l}(k)$. To know its marginal probability density functions (pdf), the principle of maximum entropy [30] is applied to its real and imaginary part respectively. This principle consists of determining the pdf that maximizes the entropy from a finite set of known expectations. This approach is relevant in our context since it provides a pdf model only from the knowledge of some moments that are easy to estimate. Basically, entropy maximization creates a model for us out of the available information [30]. Moreover, choosing the distribution with the greatest entropy avoids the arbitrary introduction or assumption of information that is not available. To obtain the model, the pdf that maximizes the entropy is constrained with measured moments of different orders [31] and then compared to the empirical pdf using the Kullback-Leibler (KL) divergence [32]. The Kullback-Leibler divergence provides a measure of the difference between two probability distributions, similar distribution having a divergence of 0 .

TABLE II

EXAMPLE OF MEASURED KULLBACK-LEIBLER DIVERGENCES FOR VARIOUS CONSTRAINTS ON THE MODEL

\begin{tabular}{|l|c|c|c|c|c|c|}
\hline Moment orders & 0 & $\leq 1$ & $\leq 2$ & $\leq 3$ & $\leq 4$ & $\leq 5$ \\
\hline KL divergence & 0.64 & 0.62 & $8.810^{-3}$ & $7.510^{-3}$ & $7.510^{-3}$ & $6.310^{-3}$ \\
\hline
\end{tabular}

Table II shows an example of a Kullback-Leibler divergence measured on a tap of channel probed in the Atlantic Ocean. On this representative example, it can be seen that the Kullback-Leibler measure converges to stable low values as soon as the second order constraint is applied to the model of maximum entropy. Applying second order constraints to the model of maximum entropy leads to a Gaussian distribution. Therefore, this indicates that the marginal distributions of the $w_{l}(k)$ can be well approximated by zeromean Gaussians. Moreover, since the joint pdf that maximizes the entropy given normal marginal pdfs is normal [33], $w_{l}(k)$ can be modeled as a strictly stationary bivariate Gaussian process. As an illustration, a joint histogram of the real and the imaginary part of a random component is shown in Figure 8.

To confirm the Gaussian result, a Kolmogorov-Smirnov [34] test is applied to the envelope of the $w_{l}(k)$. From the model, $\left|w_{l}(k)\right|$ must follow a Rayleigh distribution. $96 \%$ of the tested taps pass the test with a significance level greater than $5 \%$. We recall that a result is said to be statistically significant if it is unlikely to have occurred by chance. $5 \%$ is a conventional significance level [35]. The fact that a consistent pdf model is found across the different taps suggests that the trend stationary model assumed in (A1) and (A2) is valid. Consequently, each tap $g_{l}(k)$ can be modeled as a Rice process with a slow 
time varying mean component. In addition, as shown in Figure 9, the power ratio (Rice factor) between the trend $d_{l}(k)$ and the random component $w_{l}(k)$ is delay dependent and tends to zero (Rayleigh fading) for the most delayed paths. In the case of a null Rice factor, $g_{l}(k)=w_{l}(k)$.

Given that $w_{l}(k)$ is a Gaussian process, it is fully characterized by its first and second order statistics. Measurements and statistical tests indicate that it is a zero-mean process but it seems difficult to find a general parametric model for its second order statistics, represented by its Doppler power spectrum [12]. Observations (not shown here) seem to indicate that Doppler spectra are different from a channel to another. In addition, to get a full parametric channel model we would also need to find a parametric model for the correlation between taps as well, which seems quite elusive. However, as shown in the next section, in the context of stochastic replay it is possible to draw new realizations of the processes $w_{l}(k)$ while keeping their intrinsic second order statistics without the need for a full parametric model.

\section{STOCHASTIC REPLAY OF MEASURED IMPULSE RESPONSES}

The stochastic replay relies on the postulate that a communication channel probed at a given location over a given time window is an observation of an underlying random process. According to the previous section, the randomness of each channel tap is expressed by $w_{l}(k)$. Consequently, the vector $\left[w_{0}(k), \cdots, w_{L}(k)\right]$ is considered as a single realization of a multivariate random process $\Omega(k)$. The statistical analysis of section III states that $\Omega(k)$ is a zero-mean stationary Gaussian random process. The Rice method detailed in [36], suggests that from the observation, a multi-variate complex random Gaussian process $\Lambda(k) \triangleq\left[\lambda_{0}(k), \cdots, \lambda_{L}(k)\right]$ can be generated as follows

$$
\lambda_{l}(k)=\frac{1}{\sqrt{N}} \sum_{n=0}^{N-1} W_{l}(n) e^{2 i \pi n k / N} e^{i \theta_{l}(n)}, \forall 0 \leq l \leq L
$$

where $W_{l}(n)=\frac{1}{\sqrt{N}} \sum_{k=0}^{N-1} w_{l}(k) e^{-2 i \pi n k / N}, \theta_{l}(n)$ is an i.i.d random variable and $N$ is the observation window length. In our experiments, $N$ is large enough (from 240 to more than 15000, see section II-B) to invoke the central limit theorem and therefore guarantee the Gaussian distribution of $\lambda_{l}(k)$. Since $w_{l}(k)$ is a realization of a zero-mean process, $\theta_{l}(n)$ is chosen to be uniformly distributed in $(0,2 \pi]$.

Zero-mean Gaussian processes are fully characterized by their second order statistics or "color". The color of $\Omega(k)$ is given by its correlation matrix (or equivalently by its spectral density matrix) defined as

$$
\Gamma(u)=\left[\begin{array}{cccc}
\gamma_{00}(u) & \gamma_{01}(u) & \cdots & \gamma_{0 L}(u) \\
\gamma_{10}(u) & \gamma_{11}(u) & \cdots & \gamma_{1 L}(u) \\
\vdots & \vdots & \ddots & \vdots \\
\gamma_{L 0}(u) & \gamma_{L 1}(u) & \cdots & \gamma_{L L}(u)
\end{array}\right]
$$


where $\gamma_{l p}(u)=\mathbb{E}\left[\omega_{l}(k) \omega_{p}^{*}(k-u)\right]$. If the process is assumed to be ergodic then we have

$$
\gamma_{l p}(u)=\lim _{N \rightarrow+\infty} \frac{1}{2 N+1} \sum_{k=-N}^{N} w_{l}(k) w_{p}^{*}(k-u) .
$$

The usual WSSUS assumption would assume that $\gamma_{l p}(u)=0, \forall l \neq p$ but in our case this assumption is relaxed in order to be as close as possible to the true ocean processes. Reflections on a same physical body or delay/Doppler leakage caused by band- or time-limited transmitted data can indeed induce correlations between multipath components. In most cases, simulations of correlated multivariate random processes is quite complex and computationally costly [37], [38]. In [15], the authors bypass this difficulty by assuming that the channel scattering function [12] is separable in delay and Doppler. This separability thus reduces the correlations to a product of a temporal and a spatial correlation factor. Whereas this assumption may be valid in some specific environments such as the Baltic sound channel depicted in [15], it is not appropriate to the measures we collected in the Atlantic ocean and the Mediterranean sea.

In order to generate a multi-variate process $\Lambda(k)$ with a color similar to the one of the true process $\Omega(k)$, we suggest to create some dependence between the random phase shifts $\theta_{l}(n)$ introduced in Eq. (11). The correlation matrix elements of $\Lambda(k)$ are expressed as

$$
\begin{aligned}
\mathbb{E}\left[\lambda_{l}(k) \lambda_{p}^{*}(k-u)\right]=\frac{1}{N}( & \sum_{n=0}^{N-1} W_{l}(n) W_{p}^{*}(n) e^{2 i \pi u \frac{n}{N}} \cdot \mathbb{E}\left[e^{i\left(\theta_{l}(n)-\theta_{p}(n)\right)}\right] \\
& \left.+\sum_{n=0}^{N-1} \sum_{\substack{m=0 \\
m \neq n}}^{N-1} W_{l}(n) W_{p}^{*}(m) e^{2 i \pi \frac{k(n-m)+m u}{N}} \cdot \mathbb{E}\left[e^{i\left(\theta_{l}(n)-\theta_{p}(m)\right)}\right]\right)
\end{aligned}
$$

By choosing $\theta_{l}(n)=\theta_{p}(n)$ and i.i.d $\left\{\theta_{l}(n)\right\}_{n}$ it comes that

$$
\begin{aligned}
\mathbb{E}\left[e^{i\left(\theta_{l}(n)-\theta_{p}(n)\right)}\right] & =1 \\
\mathbb{E}\left[e^{i\left(\theta_{l}(n)-\theta_{p}(m)\right)}\right] & =0, m \neq n .
\end{aligned}
$$

Eq. (14) then simplifies to

$$
\mathbb{E}\left[\lambda_{l}(k) \lambda_{p}^{*}(k-u)\right]=\frac{1}{N} \sum_{n=0}^{N-1} W_{l}(n) W_{p}^{*}(n) e^{2 i \pi u \frac{n}{N}} .
$$

Thanks to the Plancherel-Parseval theorem, this can also be written as

$$
\mathbb{E}\left[\lambda_{l}(k) \lambda_{p}^{*}(k-u)\right]=\frac{1}{N} \sum_{k=0}^{N-1} w_{l}(k) w_{p}^{*}\left([k-u]_{N}\right)
$$

where $[.]_{N}$ denotes the modulo $N$ operator. $\mathbb{E}\left[\lambda_{l}(k) \lambda_{p}^{*}(k-u)\right]$ proves to be a very good approximation of $\gamma_{l p}(u)$ in our context. Indeed, measurements performed on the various $w_{l}(k)$ indicate that their coherence 
times are less than $600 \mathrm{~ms}$ (resp. $2 \mathrm{~s}$ ) for a 50\% (resp. 10\%) correlation level. This duration is very short compared to the duration of the available observations that ranges from 30 to $160 \mathrm{~s}$. Therefore, given that $([39], \text { pp. 195) })^{3}$

$$
\frac{1}{N} \sum_{n=0}^{N-1} \mathbb{E}\left[w_{l}(k) w_{p}^{*}\left([k-u]_{N}\right)\right]=\left\{\begin{array}{cll}
\frac{N-u}{N} \gamma_{l p}(u) & \text { for } \quad 0 \leq u \leq \frac{N}{2}, \\
\frac{u}{N} \gamma_{l p}(N-u) & \text { for } \quad \frac{N}{2} \leq u \leq N .
\end{array}\right.
$$

and that the correlation is only significant for small $u$, we can conclude that Eq. (18) is the expression of a weakly biased estimator of $\gamma_{l p}(u)$. Consequently, the true random process $\Omega(k)$ that underlies the observation can be well approximated by the process $\Lambda(k)$. Also, notice that our model allows the inphase and quadrature components of each channel tap to be mutually correlated which is equivalent to considering asymmetrical Doppler power spectral densities. The overall procedure of the channel stochastic replay can be summarized by the pseudo-code detailed in Algorithm 1. The channel model has a low computational cost since it is mainly based on discrete Fourier transforms. Only the EMD is relatively costly but it has just to be computed once and not at each realization.

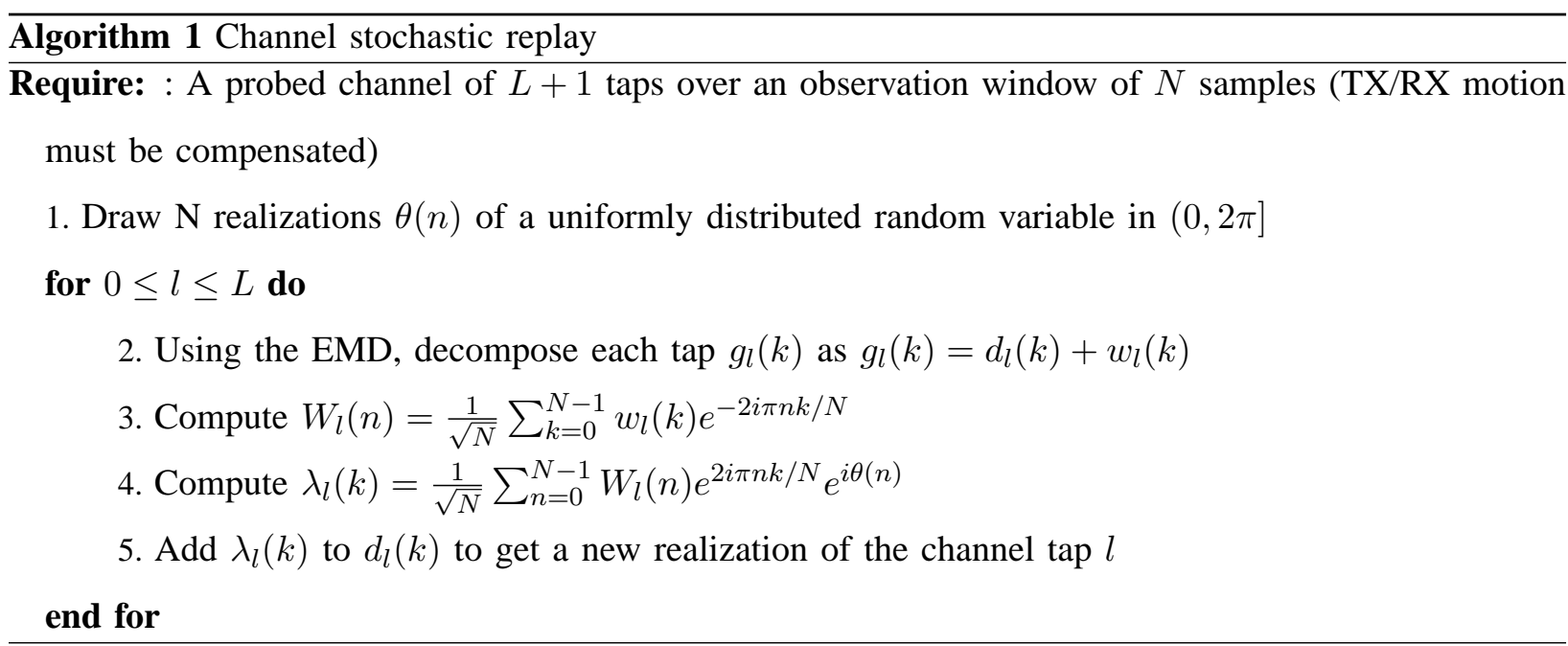

Note that non wide-sense stationary nature of the channel taps limits the stochastic replay to the duration of the original probed channel.

\section{ILLUSTRATIONS}

Stochastic replay of probed channels is of great interest to fully exploit sea experiments under controlled and reproducible laboratory conditions. From an impulse response recorded at sea, it is thus possible to

\footnotetext{
${ }^{3}$ To get this result, the correlation is assumed to be negligeable for $u>N / 2$.
} 
compute statistics and/or to test a communication system in various conditions. Through two case studies, we hereafter illustrate the benefits of channel replay for communication system design and validation.

\section{A. Fading statistics}

During the design phase of communication systems, the selection of error-correcting codes and interleavers is primarily driven by burst error statistics. These statistics are commonly given by the level crossing rate (LCR) and the average fade duration (AFD) that provide a useful means of characterizing the severity of the fading over time [16]. The LCR is defined as the rate at which the envelope $r(k)=\left|\sum_{l} g_{l}\left(k-\left\lfloor l T_{s 2} / T_{s 1}\right\rfloor\right)\right|$ crosses a specified level $\rho$ in the positive slope ( $\lfloor$.$\rfloor denotes the flooring$ operator). In the discrete-time setting, this can be expressed in terms of probability as

$$
\mathrm{LCR}=\frac{\mathrm{P}[r(k) \geq \rho, r(k-1)<\rho]}{T_{s 1}} .
$$

The AFD is defined as the average time that the fading envelope remains below a specified level after crossing that level in a downward direction

$$
\mathrm{AFD}=\frac{\mathrm{P}[r(k)<\rho]}{\mathrm{LCR}} .
$$

Figures 10 (a) and (b) highlight the benefits of stochastic replay by showing the LCR and the AFD estimated on the Mediterranean channel (b) shown in Figure 2. The solid lines represent the statistics measured on the original impulse response, and the dashed lines the statistics estimated on 1000 channel realizations using the stochastic replay. As a reference and to show the relative importance between the trend and the random component, the statistics of a "virtual" channel corresponding the original one without its random components are also displayed.

Despite the presence of two relatively stable and powerful taps at $\tau \approx 10 \mathrm{~ms}$ and $\tau \approx 20 \mathrm{~ms}$ (see Figure 2-(b)), we can observe that the random components greatly influence the metrics used for validation. The fluctuation range of the envelope is restricted to -2.5 to $2.5 \mathrm{~dB}$ for the channel including the trends only whereas the envelope ranges from -18 to $5 \mathrm{~dB}$ for the original channel with both components (pseudodeterministic and random). This can also be observed on Figure 11-(b), where the channel envelopes are plotted as a function of time.

Figures 10 (a) and (b) also show that measurements on the original impulse response recorded at sea provide estimates of the fading statistics with a relatively large variance. This impulse response indeed corresponds to a single realization of a random process over a finite time window of 28 seconds. However, thanks to the stochastic replay method, this variance can be drastically reduced by drawing a large number 
of channel realizations (1000 in our simulation). This is particularly relevant for deep fades (i.e. low $\rho$ ) that usually strongly affect the performance of communications systems.

\section{B. Reception performance}

During communication system validation, the stochastic channel replay approach can prove to be a good complementary test method to field trials. While field trials guarantee realistic testing conditions, they are limited to a narrow validation scope since they correspond to "snapshots" of specific operational environments. A great advantage of the channel replay approach is that the contributions of physical phenomena on the communication systems performance can be, to a certain extent, assessed independently. For instance, from the measured impulse response it is possible to test communications systems for various configurations (baud rate, constellation etc.) in various environments (noise type and/or power etc.).

To illustrate the relevance of channel replay for system validation, we have measured the performance of a QPSK communication link for several signal-to-noise ratios. The baud rate was set to 1000 to match the bandwidth of the probed channel. The receiver is here made of an adaptive data-aided intersymbol interference canceler (IC) [40] with joint phase tracking. Filters coefficients are updated by a data-aided least-mean square algorithm (LMS-DA) and phase tracking is performed by a second order PLL. As the objective here is not to tune the receiver to obtain the best possible performance but to exhibit some possibilities offered by the channel replay, the IC is data-aided over the entire input signal duration and takes into account the causal as well as the anti-causal interference. The IC is initialized by centering the strongest peak of the power delay profile in the feedforward filter at the start of the equalization process [15].

Similarly to Section V-A, we have performed three different kinds of simulation that are shown in Figure 11-(a). First, we have a measured the bit-error rate (BER) as a function of Eb/N0 using the original probed channel (solid line), which corresponds to a simple or a deterministic replay of the impulse response shown in Figure 2-(b). We then have performed the same kind of assessment using the stochastic replay mode and averaging the results over 1000 realizations (dashed line). The results obtained with the original channel without its random components are also displayed in order to quantify the relative importance of the pseudo-deterministic components on the BER.

In the first place, Figure 11-(a) points out the performance loss induced by the fast fluctuations of the channel. Fast fluctuations are more difficult to track for the receiver than slow fluctuations, and, as shown in Figure 11-(b), they can lead to deep fades when the scattered taps are combined in a destructive manner. 
Figure 11-(a) also shows that the performance obtained with a simple and a stochastic channel replay are equivalent for Eb/N0 less than $10 \mathrm{~dB}$. However, as Eb/N0 increases the BER difference between the two modes of replay gets larger. Once again, this difference is attributable to the fact that the simple replay only use a single realization of a random process. By looking at the time repartition of the errors of the simple replay mode, we noticed that for large Eb/N0, the errors, when occurred, were only concentrated around $15.5 \mathrm{~s}$ when a 15 to $17 \mathrm{db}$ fade occurs (see Figure 11-(b)). Using the stochastic replay mode, the time repartition of the errors as well as the fade amplitudes (see Figure 11-(b)) are different from a realization to another which therefore averages the BER and leads to a more accurate performance assessment.

\section{CONCLUSIONS}

The stochastic replay of channels recorded at sea proves to be a very useful way of designing and validating underwater acoustic communication systems. From a single measured impulse response, it is possible to independently evaluate the impact of various physical phenomena on the communication link with a good statistical significance level. The channel model derived in this contribution is based on the analysis of real data recorded in the Atlantic ocean and the Mediterranean sea. It has been shown that the analyzed underwater acoustic communication channels can be well modeled by trend stationary random processes (as opposed to usual wide-sense stationary processes). Moreover, providing that the duration of the recorded signal is greater than the channel coherence time, we have shown that the potential correlation between scatterers can easily be simulated without requiring a full parametric model.

\section{ACKNOWLEDGMENT}

The authors are grateful to the GESMA for providing part of the experimental data used in this paper. They are also very thankful to the anonymous reviewers for their constructive remarks that helped improve the quality of this paper.

\section{REFERENCES}

[1] L. M. Brekhovskikh and Yu P. Lysanov, Fundamentals of Ocean Acoustics, Springer-Verlag, 1991.

[2] X. Cristol, "NARCISSUS-2005: A Global Model of Fading Channel for Application to Acoustic Communication in Marine Environment," in Proc. IEEE Oceans Conf., Jun. 2005.

[3] F.-X. Socheleau, C. Laot, and J.-M. Passerieux, "Concise derivation of scattering function from channel entropy maximization," IEEE Trans. Commun, vol. 58, no. 11, Nov. 2010.

[4] J.J. Zhang, A. Papandreou-Suppappola, B. Gottin, and C. Ioana, "Time-Frequency Characterization and Receiver Waveform Design for Shallow Water Environments," IEEE Trans. Signal Process., vol. 57, no. 8, pp. 2973-2985, 2009. 
[5] M. Stojanovic, "Underwater Acoustic Communications: Design Considerations on the Physical Layer," in Proc. IEEE Conf. on Wireless On demand Network Systems and Services, Jan. 2008.

[6] X. Geng and A. Zielinski, “An eigenpath underwater acoustic communication channel model," in Proc. IEEE Oceans Conf., Oct. 1995.

[7] R. Galvin and R.E.W. Coats, "A stochastic underwater acoustic channel model,” in Proc. IEEE Oceans Conf., Sep. 1996.

[8] C. Bjerrum-Niese and R. Ltzen, "Stochastic Simulation of Acoustic Communication in Turbulent Shallow Water," IEEE J. Ocean. Eng., vol. 25, no. 4, 2000.

[9] A. Essebbar, G. Loubet, and F. Vial, "Underwater Acoustic Channel Simulations for Communication," in Proc. IEEE Oceans Conf., Sep. 1994.

[10] G. Xie, J. Gibson, and L. Diaz-Gonzalez, "Incorporating Realistic Acoustic Propagation Models in Simulation of Underwater Acoustic Networks: A Statistical Approach ,” in Proc. IEEE Oceans Conf., Sep. 2006.

[11] “Ocean acoustics library," http://oalib.hlsresearch.com/.

[12] P. A. Bello, "Characterization of randomly time-variant linear channels," IEEE Trans. Commun. Systems, vol. 11, no. 4, pp. 360-393, 1963.

[13] F.-X. Socheleau, J.-M. Passerieux, and C. Laot, "Characterisation of Time-Varying Underwater Acoustic Communication Channel with Application to Channel Capacity," in Proc. Underwater Acoustic Measurement: Technologies and Results, Jun. 2009.

[14] P. Qarabaqi and M. Stojanovic, "Statistical Modeling of a Shallow Water Acoustic Communication Channel," in Underwater Acoustic Measurement: Technologies and Results, Jun. 2009.

[15] P. van Walree, P. Jenserud, and M. Smedsrud, "A Discrete-Time Channel Simulator Driven by Measured Scattering Functions," IEEE J. Sel. Areas Commun., vol. 26, no. 9, pp. 1628-1637, 2008.

[16] M. Patzold, Mobile fading channels, Wiley, 2002.

[17] H. Artes, G. Matz, and F. Hlawatsch, "Unbiased Scattering Function Estimators for Underspread Channels and Extension to Data-Driven Operation," IEEE Trans. Signal Process., vol. 52, no. 5, pp. 254-264, 2004.

[18] T. H. Eggen, A. B. Baggeroer, and J. C. Preisig, "Communication Over Doppler Spread Channels Part I: Channel and Receiver Presentation," IEEE J. Ocean. Eng., vol. 25, no. 1, 2000.

[19] N. E. Huang, Z. Shen, S. R. Long, M.L. Wu, H. H. Shih, Q. Zheng, N. C. Yen, C. C. Tung, and H. H. Liu, "The empirical mode decomposition and Hilbert spectrum for nonlinear and nonstationary time series analysis," Proc. R. Soc. London A, vol. 454, pp. 903-995, 1998.

[20] P. Fan and M. Darnell, Sequence Design for communications applications, Research Studies Press Ltd, 1996.

[21] A. Goalic, J. Trubuil, and N. Beuzelin, "Long Range Real-Time Underwater Acoustic Communication at Low Bit Rate with Channel Coding protection," in IEEE Military Communications Conference, Nov. 2008.

[22] B.S. Sharif, J. Neasham, O.R. Hinton, and A.E. Adams, "A Computationally Efficient Doppler Compensation System for Underwater Acoustic Communications," IEEE J. Ocean. Eng., vol. 25, no. 1, 2000.

[23] G. Eynard and C. Laot, "Blind Doppler Compensation Techniques for single Carrier Digital Underwater Communications," in Proc. IEEE Oceans Conf., Sep. 2008.

[24] P. Borgnat, P. Flandrin, P. Honeine, C. Richard, and J Xiao ; "Testing Stationarity With Surrogates: A Time-Frequency Approach," IEEE Trans. Signal Process., vol. 58, no. 7, pp. 3459-3470, 2010.

[25] J.G. Proakis, Digital Communications, McGraw-Hill, 2001. 
[26] G. Rilling, P. Flandrin, P. Goncalves, and J.M. Lilly, "Bivariate Empirical Mode Decomposition,” IEEE Signal Process. Lett., vol. 14, no. 12, pp. 936-939, 2007, software available at http://perso.ens-lyon.fr/patrick.flandrin/emd.html.

[27] S.R. Massel, Ocean Surface Waves: Their Physics and Prediction, World Scientific Pub Co Inc, Hanover, 1996.

[28] G. Rilling, P. Flandrin, and P. Goncalves, "On Empirical Mode Decomposition and its algorithms," in IEEE-EURASIP Workshop on Nonlinear Signal and Image Processing, Jun. 2003.

[29] A. Radosevic, J. G. Proakis, and M. Stojanovic, "Statistical Characterization and Capacity of Shallow Water Acoustic Channels," in Proc. IEEE Oceans Conf., May 2009.

[30] E.T. Jaynes, "Information Theory and Statistical Mechanics," Physical review, vol. 106, no. 4, pp. 620-630, 1957.

[31] A. M. Djafari, “A Matlab Program to Calculate the Maximum Entropy Distributions," http://djafari.free.fr/pdf/me91_1.pdf.

[32] T. Cover and J. Thomas, Elements of Information Theory, Wiley, 1991.

[33] H. Joe, Multivariate models and dependence concepts, Chapman/Hall, 1997.

[34] M.A. Stephens, "EDF Statistics for Goodness of Fit and Some Comparisons," Journal of the American Statistical Association, vol. 69, no. 347, pp. 730-737, 1974.

[35] S. Stigler, "Fisher and the 5\% level," Chance, vol. 21, no. 4, 2008.

[36] S. O. Rice, "Mathematical analysis of random noise," Bell Syst. Tech Journal, vol. 23, pp. 282-332, 1944.

[37] G. Deodatis, "Simulation of Ergodic Multivariate Stochastic Processes," Journal of Engineering Mechanics, vol. 122, no. 8, pp. 778-787, 1996.

[38] L. Chen and C. W. Letchford, "Simulation of Multivariate Stationary Gaussian Stochastic Processes: Hybrid Spectral Representation and Proper Orthogonal Decomposition Approach,” Journal of Engineering Mechanics, vol. 131, no. 8, pp. 801-808, 2005.

[39] J. Max and J.-L. Lacoume, Méthodes et techniques de traitement du signal, Dunod, 2004.

[40] C. Laot, R. Le Bidan, and D. Leroux, "Low-Compexity MMSE Turbo Equalization: A possible Solution for EDGE," IEEE Trans. Wireless Commun., vol. 4, no. 3, 2005.

[41] N. ur Rehman and D.P. Mandic, "Empirical Mode Decomposition for Trivariate Signals," IEEE Trans. Signal Process., vol. 58, no. 3, Mar. 2010.

[42] Z. Wu, N. E. Huang, S. R. Long, and C.-K. Peng, "On the trend, detrending, and variability of nonlinear and nonstationary time series," Proc. of the National Academy of Sciences, vol. 104, no. 38, 2007. 


\section{APPENDIX A}

\section{EMPIRICAL MODE DECOMPOSITION}

The material presented in the appendix is a compilation of works published in [19], [26], [41], [42].

The Empirical Mode Decomposition (EMD) seeks the different intrinsic modes of oscillations in any data based on the principle of local scale separation. It is designed to define a local low frequency component as the local trend $r_{0}(t)$, supporting a local high frequency component as a zero-mean oscillation or local detail $m_{0}(t)$, so that any signal $x(t)$ can be expressed as

$$
x(t)=r_{0}(t)+m_{0}(t) .
$$

By construction $r_{0}(t)$, is an oscillatory signal, and, if it is furthermore required to be locally zero-mean everywhere, it corresponds to what is referred to as an intrinsic mode function (IMF). An intrinsic mode of oscillation is called an IMF when it satisfies:

- in the whole data set, the number of extrema and the number of zero-crossings must either equal or differ at most by one

- at any point, the mean value of the envelope defined by the local maxima and the envelope defined by the local minima is zero.

All we know about $r_{0}(t)$ is that it locally oscillates more slowly than $m_{0}(t)$. We can then apply the same decomposition to it, leading to $r_{0}(t)=r_{1}(t)+m_{1}(t)$, and, recursively applying this on the $r_{i}(t)$, we get a representation of $x(t)$ of the form

$$
x(t)=r_{Q-1}(t)+\sum_{q=0}^{Q-1} m_{q}(t) .
$$

The discrimination between fast and slow oscillations is obtained through an algorithm referred to as the sifting process, which iterates a nonlinear elementary operator $\mathcal{S}$ on the signal until the local detail can be considered as zero-mean according to some stopping criterion. Given a signal $x(t)$, the operator $\mathcal{S}$ is defined by the following procedure:

1) Identify all extrema of $x(t)$.

2) Interpolate (using a cubic spline) between minima (resp. maxima), ending up with some envelope $e_{\min }(t)\left(\right.$ resp. $\left.e_{\max }(t)\right)$.

3) Compute the mean $r(t)=\left(e_{\min }(t)+e_{\max (t)}\right) / 2$.

4) Subtract from the signal to obtain $\mathcal{S}[x](t)=x(t)-r(t)$. 
If the stopping criterion is met after $n$ iterations of the sifting process, the local detail and the local trend are defined as $m_{0}(t)=\mathcal{S}^{n}[x](t)$ and $r_{0}(t)=x(t)-m_{0}(t)$. A dynamic illustration of the EMD in operation is available at http://perso.ens-lyon.fr/patrick.flandrin/emd.html.

An extension to bivariate signals is proposed in [26]. Where the EMD extracts zero-mean oscillating components, the proposed bivariate extension is designed to extract zero-mean rotating components. In order to separate the more rapidly rotating component from slower ones, the idea is once again to define the slowly rotating component as the mean of some envelope. The envelope is now a three-dimensional tube that tightly encloses the signal. The tube is obtained by projecting the bivariate signal in several directions. 


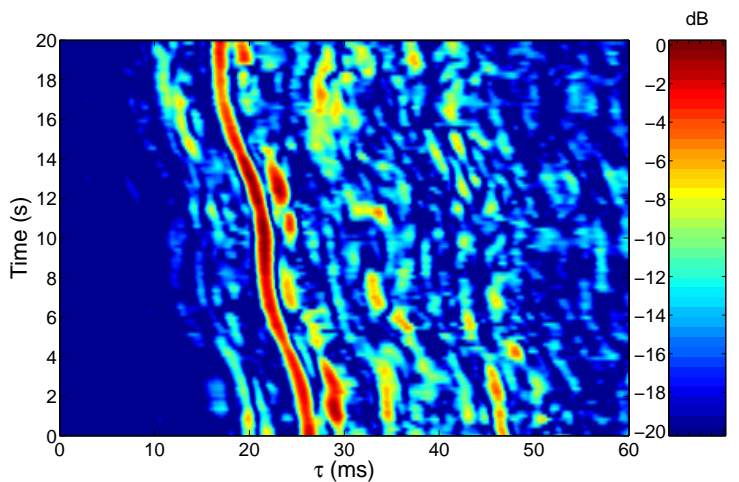

(a)

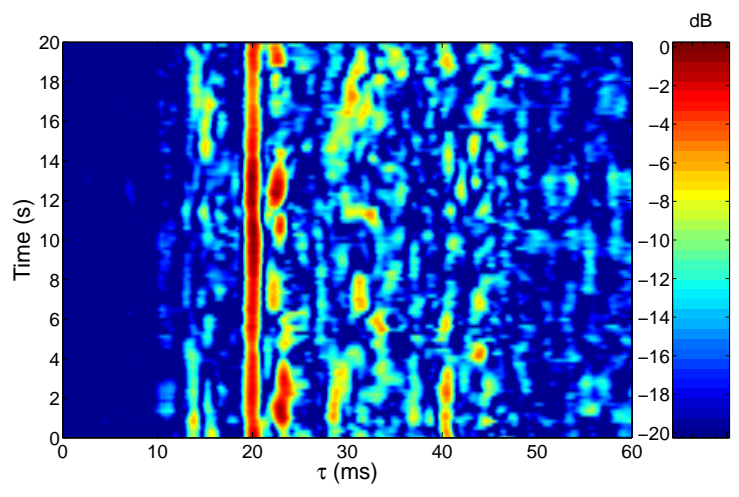

(b)

Fig. 1. Effect example of the platform motion on an estimated impulse response. (a) No Doppler mitigation, (b) Doppler mitigated. 

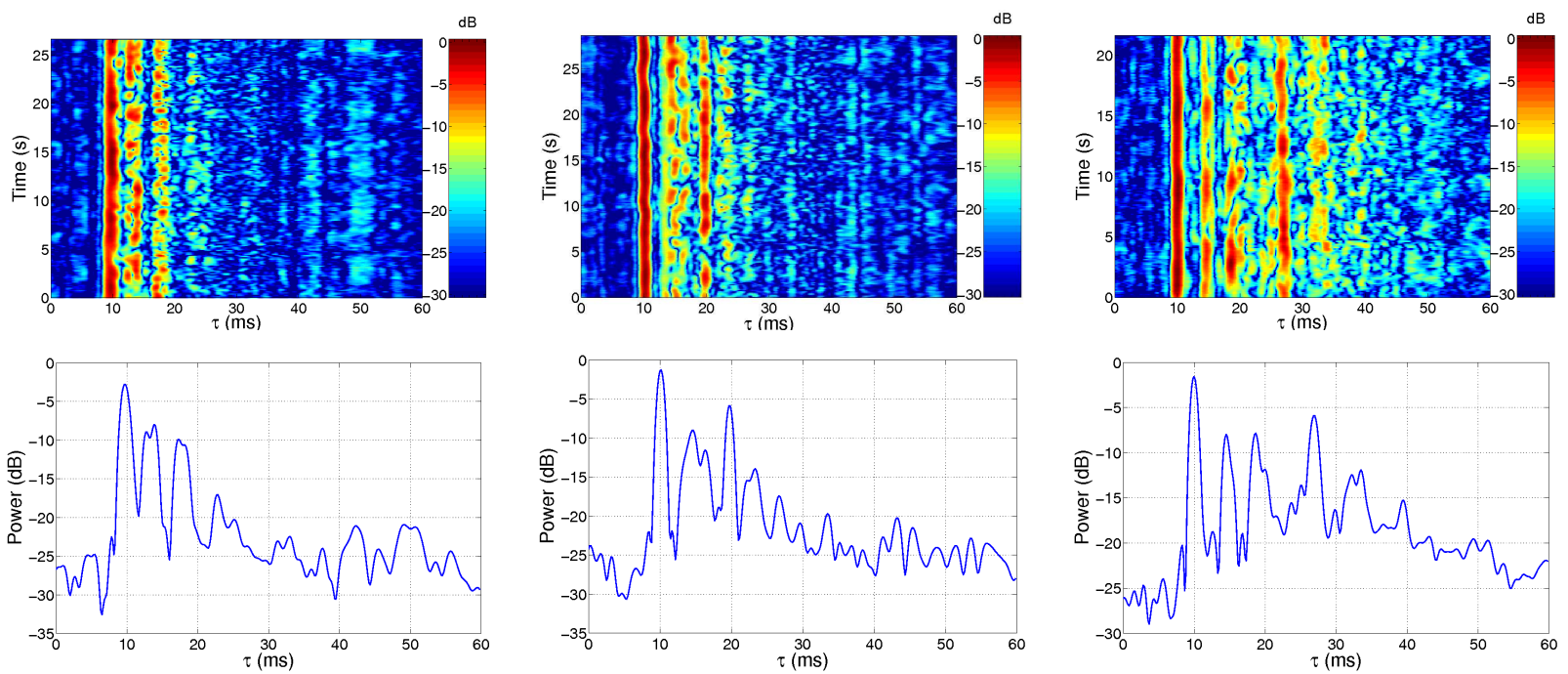

(a)

(b)
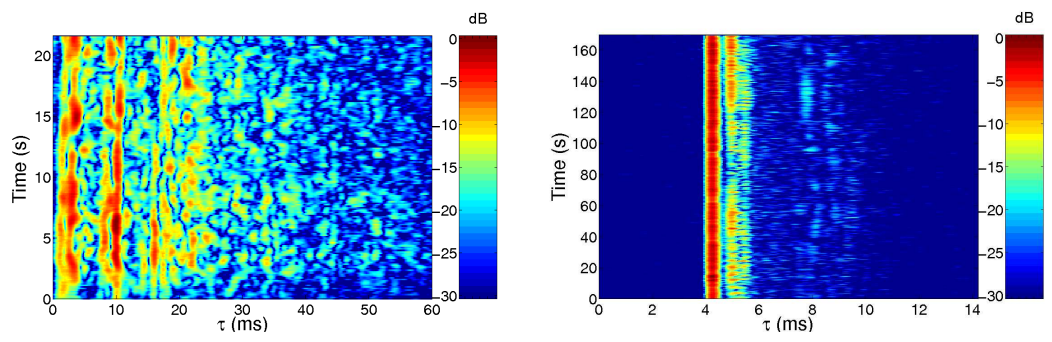

(c)
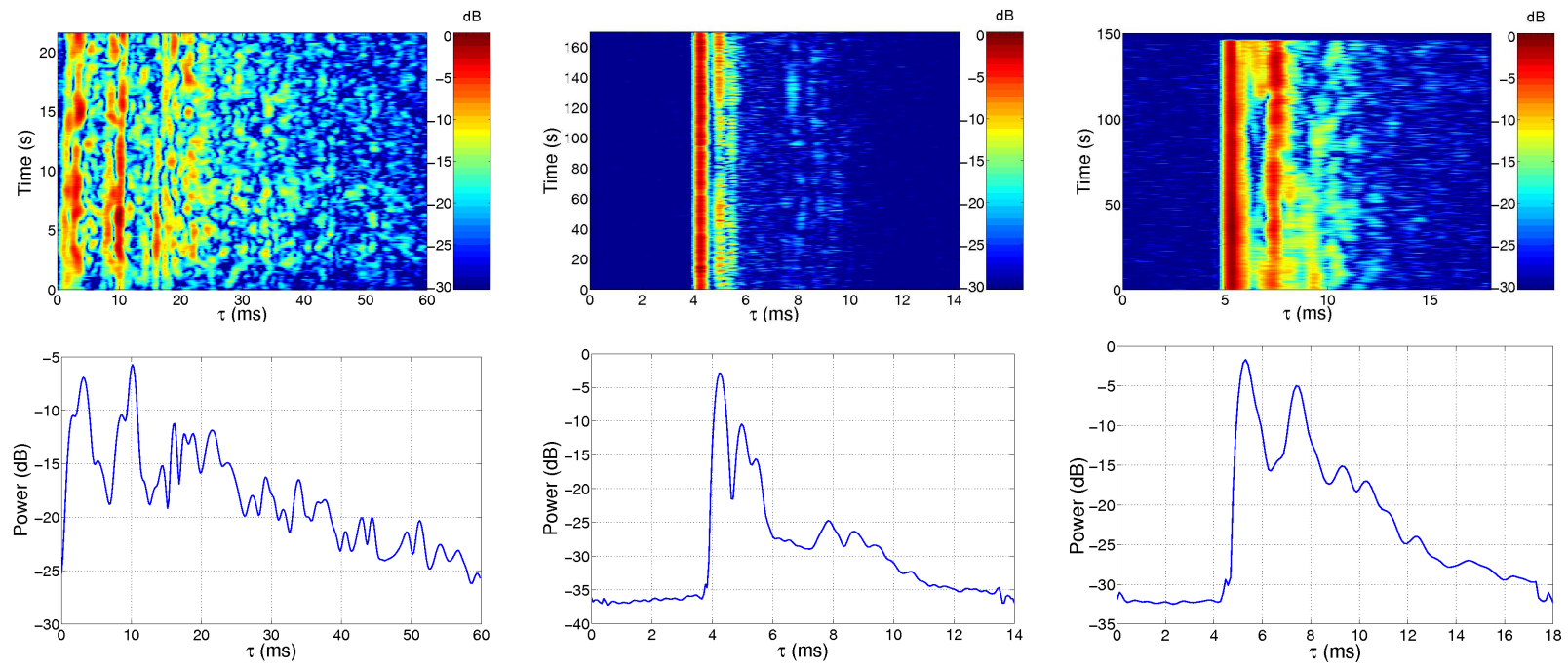

(d)

(e)

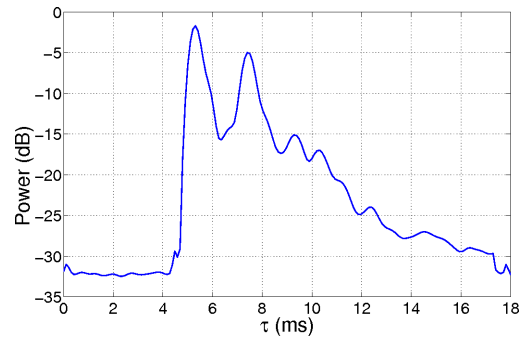

(f)

Fig. 2. Examples of measured impulse responses and power delay profiles. (a) Med. sea: $\mathrm{D}=1000 \mathrm{~m}$, (b) Med. sea: $\mathrm{D}=2500 \mathrm{~m}$, (c) Med. sea: $\mathrm{D}=5000 \mathrm{~m}$, (d) Med. sea: $\mathrm{D}=9000 \mathrm{~m}$, (e) Atl. ocean: $\mathrm{D}=1000 \mathrm{~m}$, carrier freq. $=17.5 \mathrm{kHz}$ (f) Atl. ocean: $\mathrm{D}=3000$ $\mathrm{m}$, carrier freq. $=11.2 \mathrm{kHz}$. 


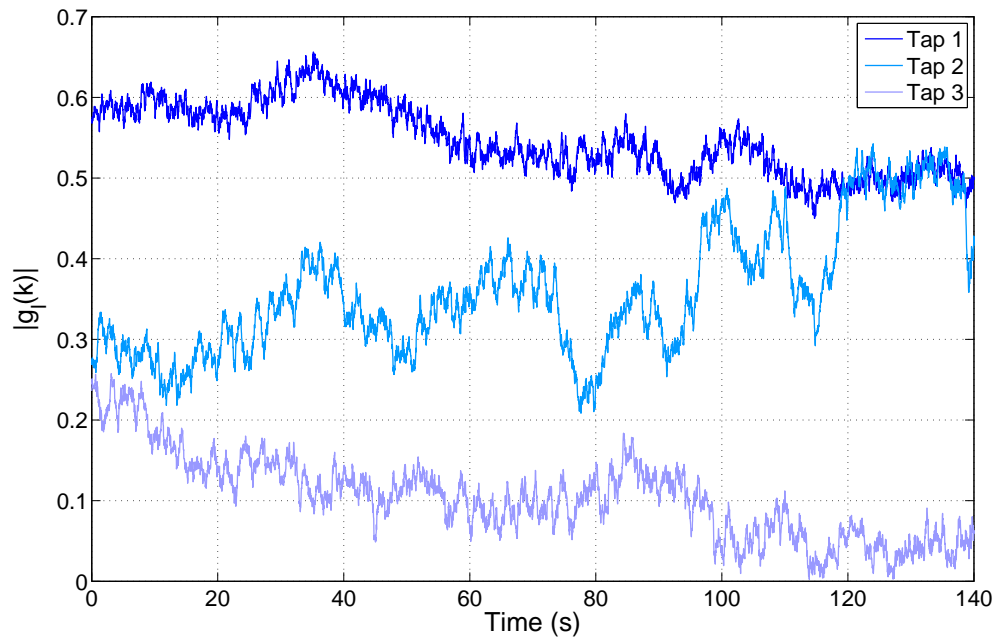

Fig. 3. Examples of the time evolution of UAC tap envelopes.
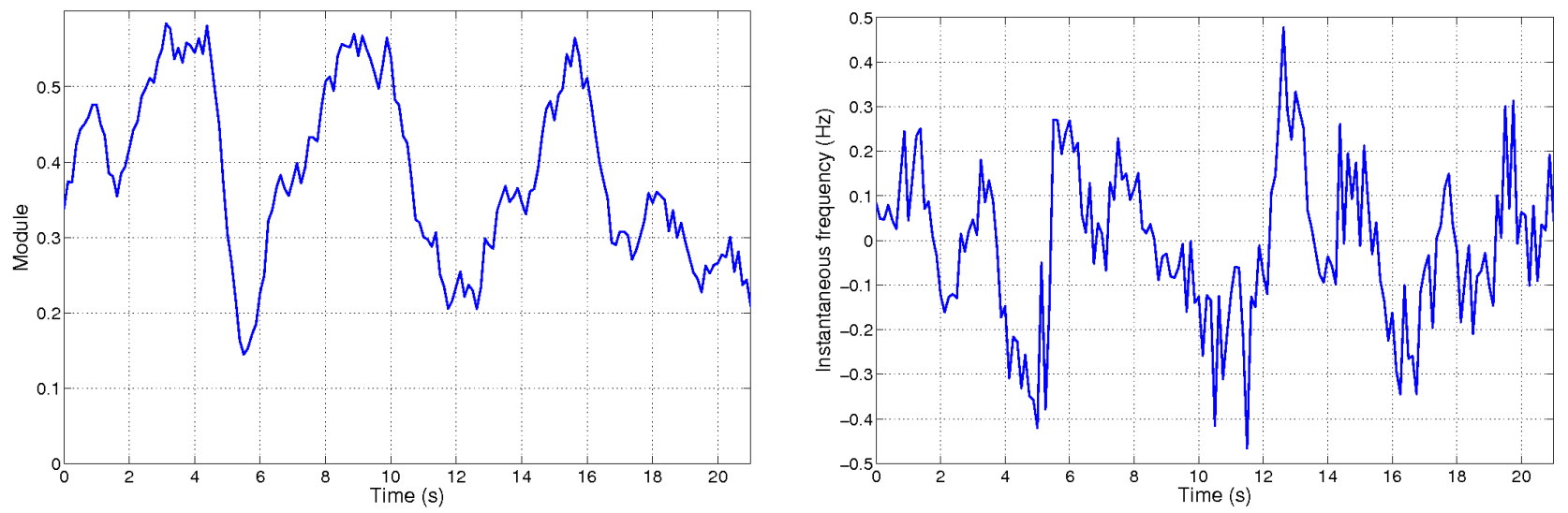

Fig. 4. Time evolution example of the module and the instantaneous frequencies of a channel tap with a powerful trend (Med. sea). 


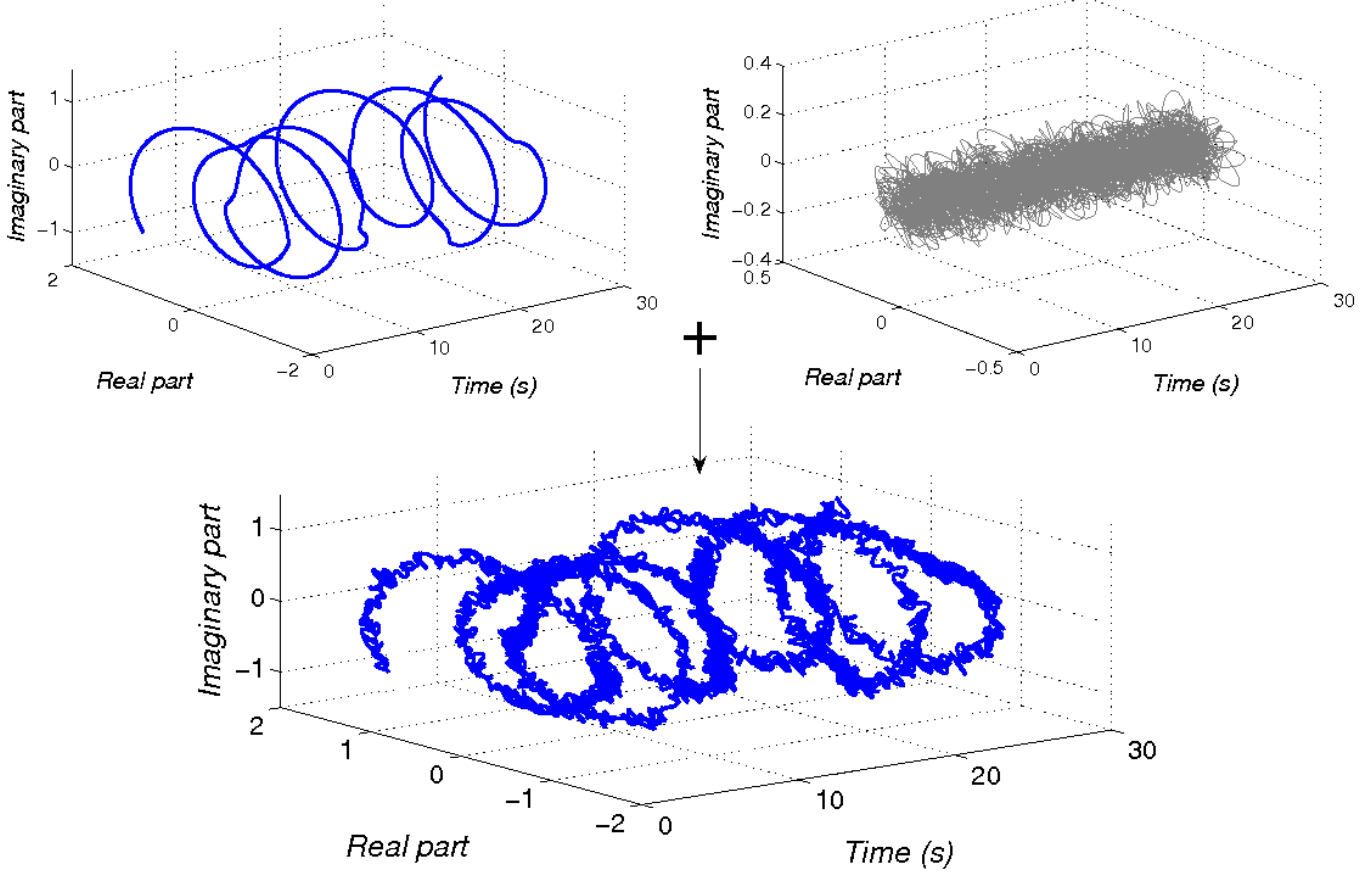

Fig. 5. Synthetic complex-valued time series. The top left figure represents the time evolution of the trend. This trend is amplitude modulated by a sine oscillating at $1 / 7 \mathrm{~Hz}$ and frequency modulated by a sine oscillating at $2 / 7 \mathrm{~Hz}$. The top right figure shows the time evolution of the random component of the time series. This component is modeled as a low-pass filtered white Gaussian noise.

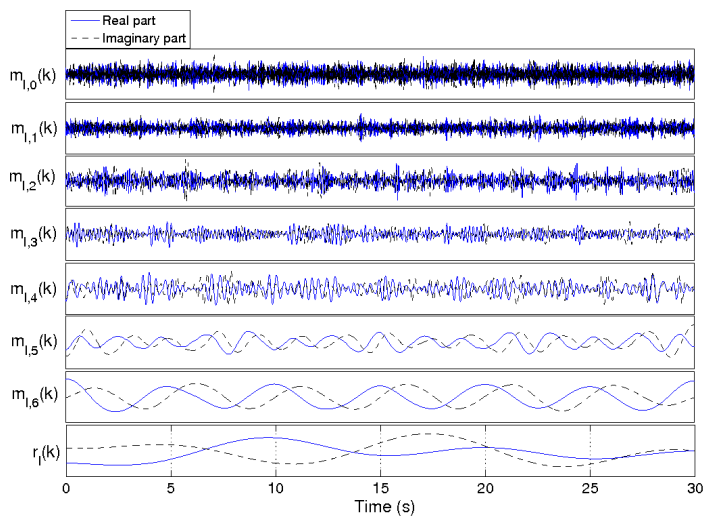

(a)

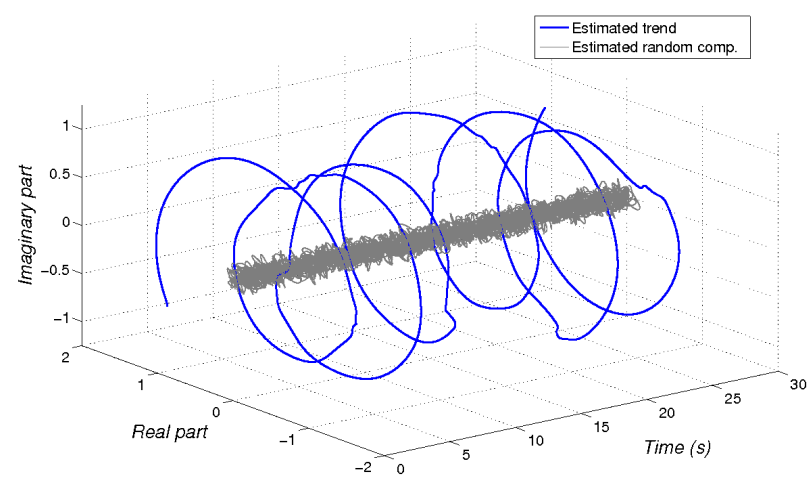

(b)

Fig. 6. (a) Intrinsic mode functions of the signal displayed on Figure 5. (b) Separation of the trend and the random component $\left(S_{l}=5\right)$. 


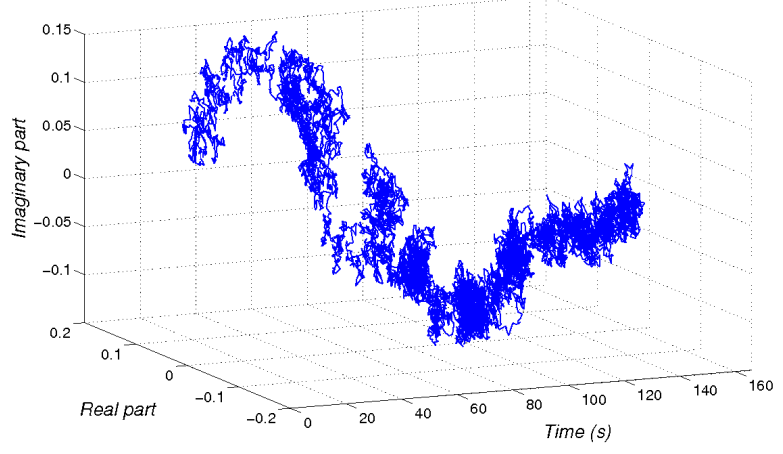

(a)

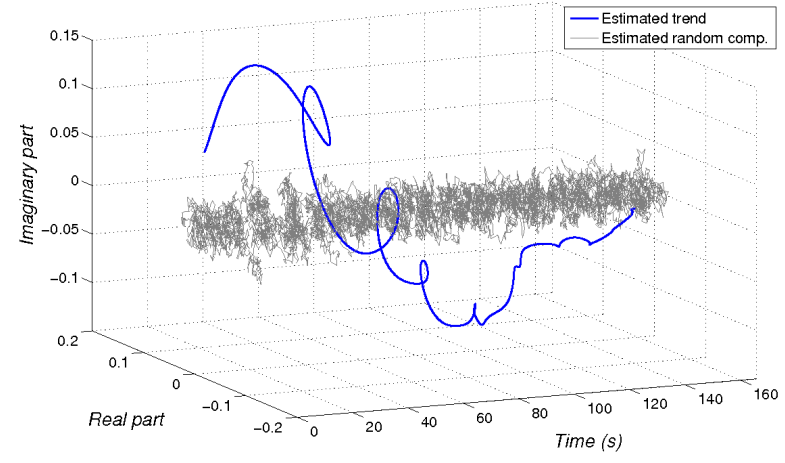

(b)

Fig. 7. Illustration of EMD filtering applied to a real channel tap. (a) Original tap, (b) Separation of the trend and the random component.

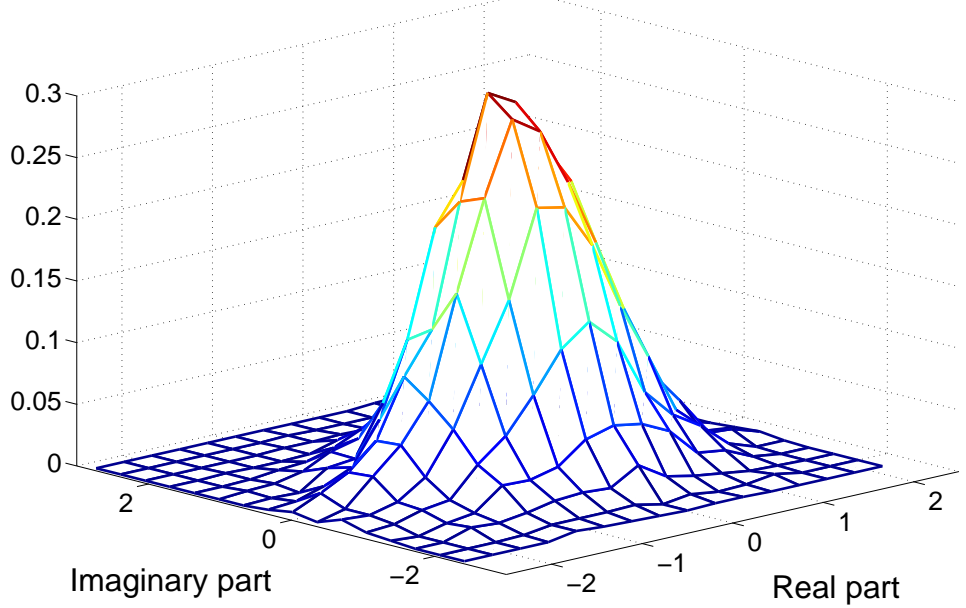

Fig. 8. Histogram of a random component. 


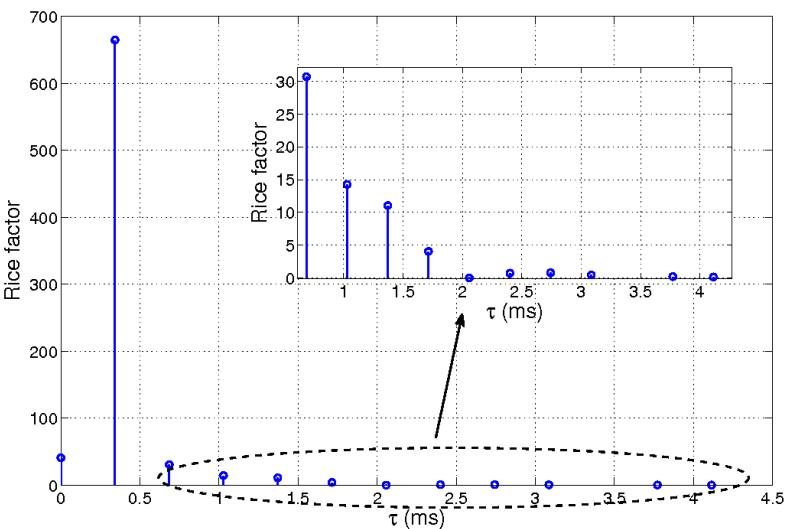

(a)

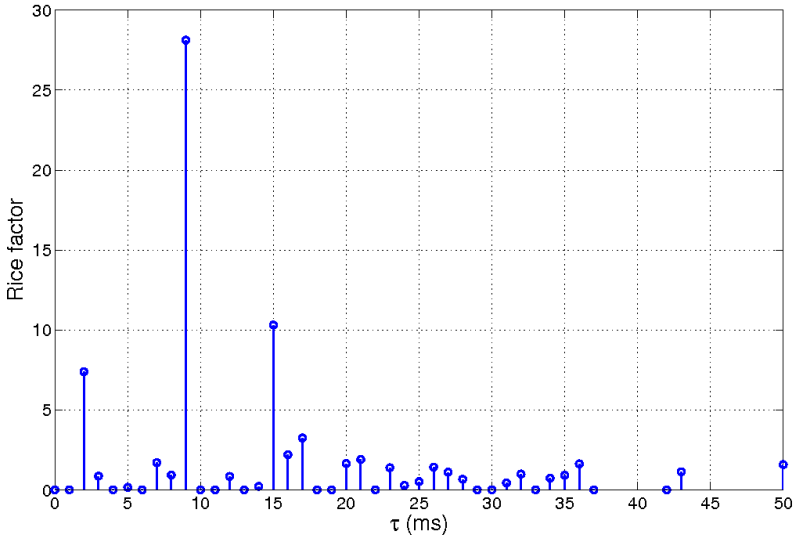

(b)

Fig. 9. Measure of the Rice factor as a function of the path delays. (a) Channel probed in the Atlantic ocean, (b) Channel probed in the Mediterranean sea.

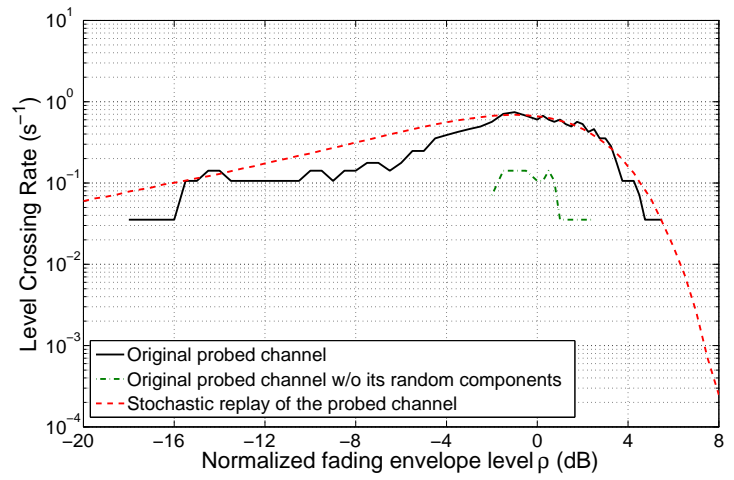

(a)

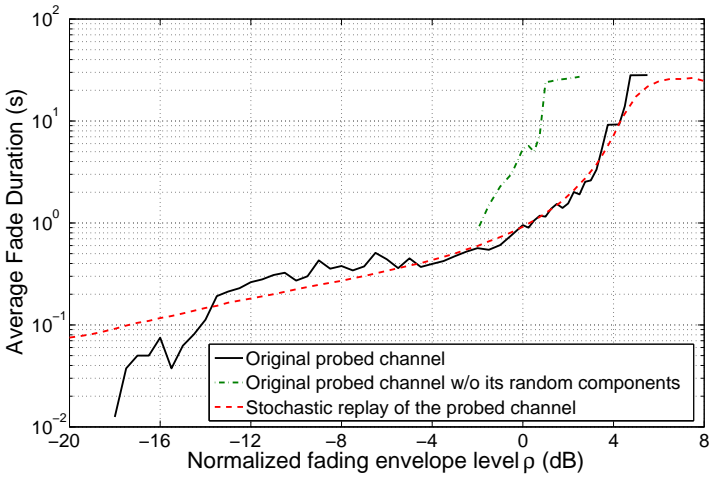

(b)

Fig. 10. Estimation results of the fading statistics: (a) LCR, (b). 


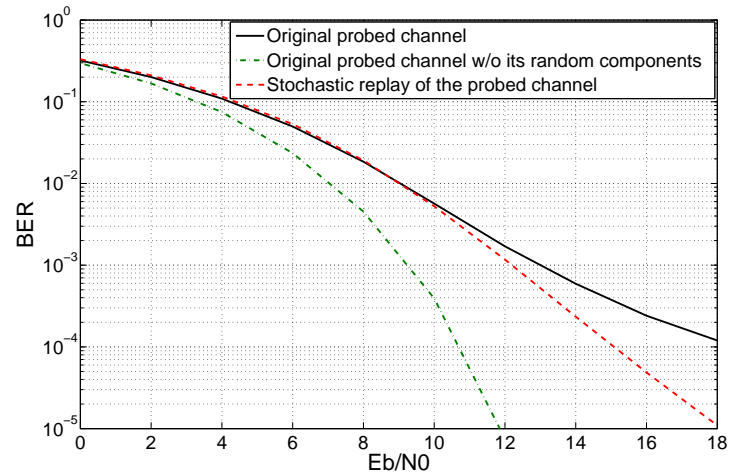

(a)

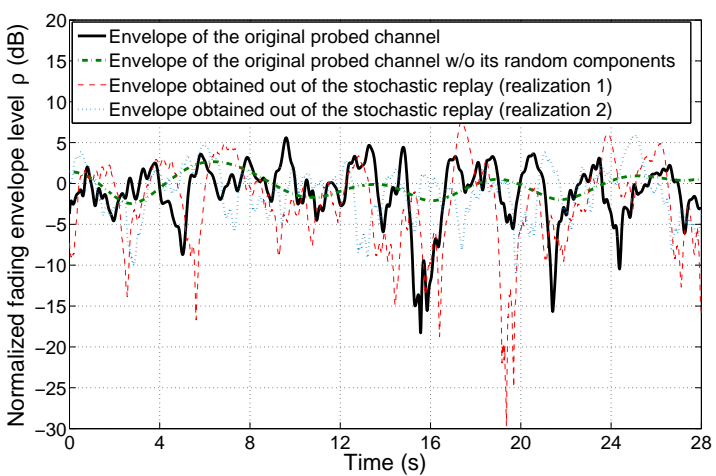

(b)

Fig. 11. Performance example of a QPSK receiver for different Eb/N0 (the simulated noise is additive white and Gaussian). (a) BER vs Eb/N0, (b) Envelope vs Time. 